\title{
Associations and indications of Ki67 expression with clinicopathological parameters and molecular subtypes in invasive breast cancer: A population-based study
}

\author{
JINZHONG SUN $^{1,2^{*}}$, CHUANG CHEN $^{1 *}$, WEN WEI $^{1 *}$, HONGMEI ZHENG $^{3}$, JINGPING YUAN $^{4,5}$, YI TU $^{1}$, \\ FENG YAO ${ }^{1}$, LIJUN WANG ${ }^{1}$, XIAOLI YAO ${ }^{1}$, JUANJUAN LI $^{1}$, YAN LI ${ }^{4}$ and SHENGRONG SUN ${ }^{1}$ \\ ${ }^{1}$ Department of Breast and Thyroid Surgery, Renmin Hospital of Wuhan University, Wuhan, Hubei 430060; \\ ${ }^{2}$ Department of General Surgery, Xiangyang Hospital, Hubei University of Medicine, Xiangyang, Hubei 431000; \\ ${ }^{3}$ Department of Breast Surgery, Hubei Cancer Hospital, Wuhan, Hubei 430079; ${ }^{4}$ Department of Oncology, \\ Zhongnan Hospital of Wuhan University, Hubei Key Laboratory of Tumor Biological Behaviors, \\ Hubei Cancer Clinical Study Center, Wuhan, Hubei 430071; ${ }^{5}$ Department of Pathology, \\ The Central Hospital of Wuhan, Wuhan, Hubei 430014, P.R. China
}

Received June 28, 2014; Accepted January 29, 2015

DOI: $10.3892 / 01.2015 .3461$

\begin{abstract}
Ki67 has potential prognostic and predictive values for breast cancer patients, and has become an important biomarker in routine clinical practice. The aims of the present study were to investigate the distribution of Ki67 expression and its correlation with other clinicopathological parameters in central China. In total, 1,259 patients with newly-diagnosed invasive breast cancer were included in the present study. The clinical information was obtained from the electronic medical records. The expression levels of Ki67, estrogen receptor (ER), progesterone receptor (PR) and human epidermal growth factor receptor 2 (HER2) were detected by immunohistochemical analysis. The associations between Ki67 scores and other prognostic factors were evaluated as continuous and categorical variables. The mean value of the Ki67 scores of all patients was $31 \%$. In total, $36 \%(456 / 1,259)$ of the patients demonstrated a low expression of Ki67. A statistically significant correlation was identified between the mean Ki67 scores and the lymph node status, tumor grade, ER, PR and HER2 status, and clinical stage or molecular subtypes (all $\mathrm{P}<0.001$ ). When Ki67 was categorized into high $(>14 \%)$ and low $(\leq 14 \%)$ level groups, the $\chi^{2}$ test was used to verify these results. The Ki67 scores demonstrated no statistically significant differences between the HER2-positive (non-luminal) and three
\end{abstract}

Correspondence to: Dr Shengrong Sun, Department of Breast and Thyroid Surgery, Renmin Hospital of Wuhan University, 238 Jiefang Road, Wuchang, Wuhan, Hubei 430060, P.R. China E-mail:sun137@sina.com

*Contributed equally

Key words: Ki67, breast cancer, molecular subtypes, clinicopathological parameters negative subtypes, with the exception of patients with a tumor size of $>2 \mathrm{~cm}(\mathrm{P}=0.02)$. In conclusion, the results revealed the presence of significant correlations between Ki67 and other clinicopathological parameters.

\section{Introduction}

Breast cancer (BC) is the most common type of cancer and the leading cause of cancer-associated mortalities (1). An improved understanding of the key molecules involved in the pathogenesis of BC is essential for personalized treatment. Ki67 is a proliferation marker that is expressed in all the phases of the cell cycle, with the exception of the $G_{0}$ phase (2-4). A number of studies have demonstrated the prognostic value of $\mathrm{Ki} 67$ in BC $(5,6)$. In addition, Ki67 levels are useful in identifying patients that are most likely to benefit from chemotherapy (7). Furthermore, changes in Ki67 levels have been used as a primary efficacy endpoint in clinical trials $(8,9)$.

At present, Ki67 is an important biomarker used in routine clinical pathological practice, with potential applications in prognosis, used to predict responses or resistance to chemotherapy and endocrine therapy, estimate residual risks in patients receiving standard therapies and as a dynamic biomarker to measure treatment efficacies in samples obtained prior to, during and subsequent to neoadjuvant therapy (10). Increasingly, Ki67 levels are used for clinical research purposes, including as a primary efficacy endpoint during clinical trials and, in certain circumstances, for clinical management. The 2011 St Gallen Expert Panel indicated that a Ki67 level of $\geq 14 \%$ distinguished luminal B from luminal A tumors in BC molecular subtyping $(11,12)$. Therefore, understanding the association of $\mathrm{Ki} 67$ with pathological characteristics (including histological grade, tumor size and lymph node metastasis) and immunohistochemical indexes [including the estrogen receptor (ER), progesterone receptor (PR) and human epidermal growth factor receptor 2 (HER2) status] is important for clinical evaluations and guiding treatment strategies. 
Until recently, limited information was available regarding the expression of Ki67 among Chinese females with BC. Furthermore, previous studies on the association between Ki67 and other clinicopathological parameters have only included small sample sizes (13-15); therefore, this association remains controversial. The aim of the present study was to investigate the distribution of Ki67 expression and its association with other clinicopathological parameters. In addition, Ki67 expression in molecular subtypes with various tumor sizes or lymph node statuses were analyzed.

\section{Materials and methods}

Patients. The present study was population-based and included a cohort of females with newly-diagnosed BC who had been treated in the breast centers of four hospitals in Wuhan, China, between June 2011 and December 2012. The study included patients from the Renmin Hospital of Wuhan University, Zhongnan Hospital of Wuhan University, Hubei Cancer Hospital and the Central Hospital of Wuhan. In total, 1,535 cases were included in the study. Only invasive BC cases were included; male patients or those with incomplete medical records were excluded. Overall, 1,259 patients were eligible to for inclusion in the this study.

Clinical information regarding age at diagnosis, ethnicity, clinical stage, pre-surgical chemotherapy treatments, type of surgery and post-surgical therapy treatments, was retrieved from the electronic medical records subsequent to Institutional Review Board approval. The tumor size and grade, nodal stage, status of molecules (ER, PR, HER2 and Ki67) and histological subtype were acquired from the pathology database. As part of the clinical work-up, these investigations were performed prospectively upon excision specimens at the time of diagnosis. According to specific criteria, all the pathological results of this study were histopathologically analyzed by two experienced histological pathologists. The classification was performed according to definitions provided by the World Health Organization (16). The tumors were graded according to the modified Nottingham grading system (17). The patients were staged according to the 2010 7th edition of the American Joint Committee on Cancer tumor-node-metastasis (TNM) staging system for BC (18). The study was approved by the Ethics Committee of Wuhan University.

ER, PR, HER 2 and Ki67 detection. Tumor samples obtained from the patients were assessed or reassessed (if the initial results were already available) by two experienced pathologists to obtain the Ki67 score, as well as the ER, PR and HER2 status. The four hospitals used the same primary antibodies.

ER and PR immunohistochemical staining was performed using a mouse monoclonal anti-human ER antibody (clone, 1D5; Dako, Glostrup, Denmark) and a mouse monoclonal anti-human PR antibody (clone, 636; Dako) at a 1:100 dilution. The cut-off value for a positive result was positive staining for ER and PR in $\geq 1 \%$ of tumor cells in 10 selected tumor sub-regions (19). The results were recorded as the percentage of positively-stained nuclei, and the intensity was graded between 0 and $3+$ as follows: i) 0 (negative result), positive staining in $<1 \%$ of the tumor cells; ii) $1+$, mildly distinct, positive staining in $\leq 25 \%$ of the tumor cells; iii) $2+$, moderately distinct, positive staining in $25 \%-50 \%$ of the tumor cells; and iv) $3+$, strong, positive staining in $>50 \%$ of the tumor cells.

HER2 immunohistochemical staining was performed using the HercepTest ${ }^{\mathrm{TM}}$ assay (Dako). The expression of HER2 was initially determined by immunohistochemistry and graded between 0 and $3+$ as follows: i) 0 (negative result), absence or presence of HER 2 in $<10 \%$ of the tumor cells; ii) $1+$ (negative result), membranous, weak and discontinuous staining in $>10 \%$ of the tumor cells; iii) 2+ (questionable result), membranous, low/moderate and continuous staining in $>10 \%$ of the tumor cells, or membranous, intense and continuous staining in $\leq 30 \%$ of the tumor cells; and iv) $3+$ (positive result), membranous, intense and continuous staining in $>30 \%$ of the tumor cells. Samples with HER2 scores of 2+ were confirmed to be HER2-negative or HER2-positive using fluorescence in situ hybridization analysis (20).

Ki67 immunohistochemical staining was performed using a mouse monoclonal anti-human Ki67 antibody (clone, MIB-1; Dako) at a 1:100 dilution. At least three fields in particular staining 'hot-spots' were selected in order to represent the spectrum of staining observed upon the initial overview of the entire section. The cancer cells in the three micrographs were manually counted (500-1,000 cells were counted), and the percentage of positively-stained cancer cells were considered to be the Ki67 score (10).

The BC cases were divided into five subtypes based on the expression levels of ER, PR and HER2, and the Ki67 proliferation index as follows: i) Luminal A subtype: ER- and/or PR-positive, HER2-negative and a low Ki67 proliferation index of $\leq 14 \%$; ii) luminal B (high Ki67) subtype: ER- and/or PR-positive, HER2-negative and a high Ki67 index (>14\%); iii) luminal B (HER2-positive) subtype: ER- and/or PR-positive, HER2-positive and any Ki67 index; iv) HER2-positive (non-luminal) subtype: ER- and PR-negative, HER2-positive and any Ki67 index; and v) triple-negative subtype: ER-, PRand HER2-negative, and any Ki67 index (21).

Statistical analysis. The correlation of Ki67 as a categorical variable was determined using the $\chi^{2}$-test, two-sample t-test or one-way analysis of variance for continuous variables. When equal variances were assumed, post hoc multiple comparisons used the least significant difference and Student-Newman-Keuls tests. When equal variances were not assumed, Dunnett's T3 and Dunnett's C tests were used. Statistical analyses were performed using SPSS version 21.0 software (SPSS Inc., Chicago, IL, USA). Two-tailed P $<0.05$ was considered to indicate a statistically significant difference.

\section{Results}

Clinicopathological features of patients and distribution of Ki67 expression. The clinicopathological characteristics of the $\mathrm{BC}$ patients are listed in Table I. All the patients were female, with a median age of 50 years (range, 20-91 years). The majority of the tumor sizes ranged between 2 and $5 \mathrm{~cm}(\mathrm{pT} 2)$ (65\%). All the patients underwent axillary dissection. In total, 673/1,259 cases (53\%) were lymph node-negative. Grade II tumors accounted for $62 \%(782 / 1,259)$ of cases, whilst the ER-, PR- and HER2-positive rates were 60\% (758/1,259), 51\% $(642 / 1,259)$ and $35 \%(439 / 1,259)$, respectively. 


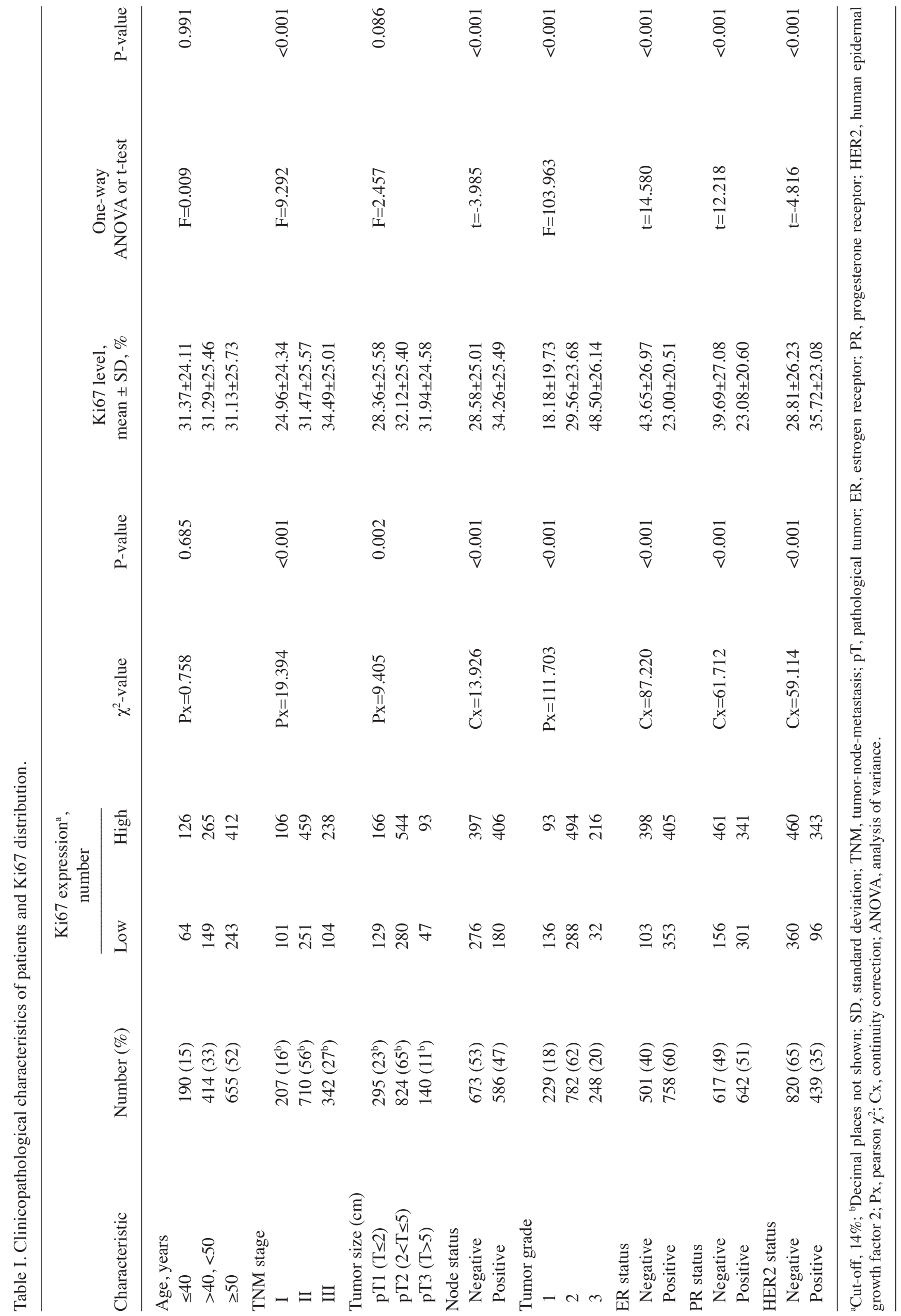


A

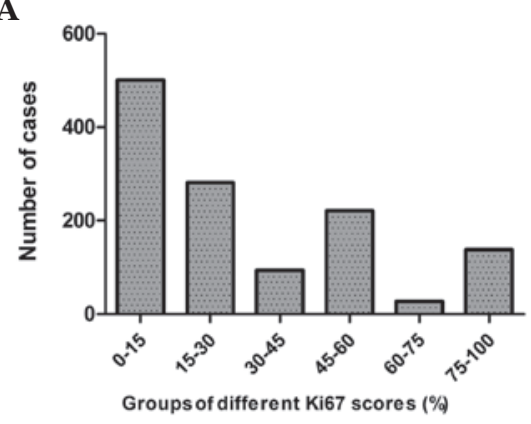

C

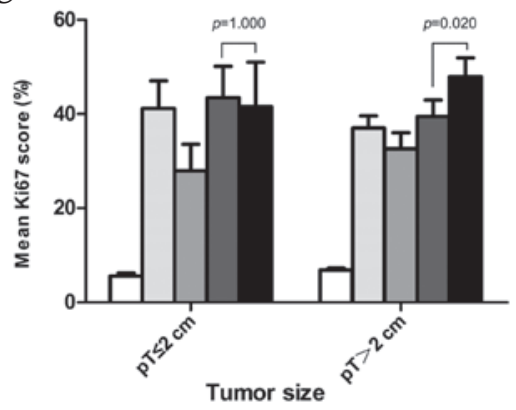

B

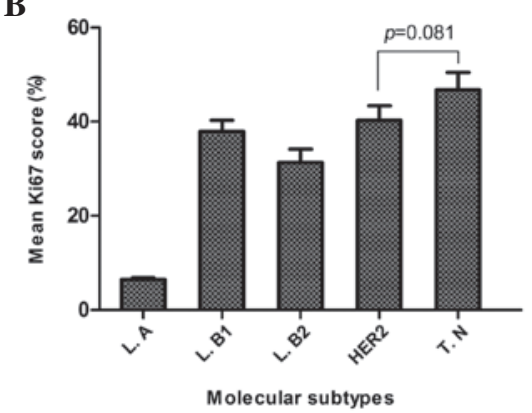

D

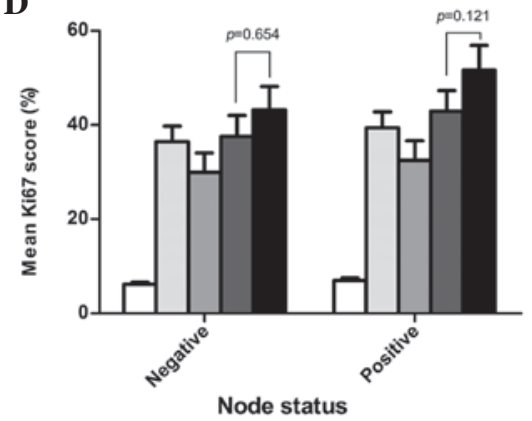

口L.A $\square$ L. B1 $\square$ L. B2 HER2 $\square$ T. N

Figure 1. Distribution of Ki67 expression in the breast cancer cases and specific molecular subtypes. (A) Number of cases in each group with different Ki67 scores. (B) Mean Ki67 scores in the different molecular subtypes. (C) Mean Ki67 scores in the molecular subtypes with different tumor sizes. (D) Mean Ki67 scores in the molecular subtypes with different node statuses. L.A, luminal A; L.B1, luminal B (high Ki67); L.B2, luminal B (HER2-positive); HER2, HER2-positive (non-luminal); T.N, triple-negative; HER2, human epidermal growth factor receptor 2; pT, pathological tumor.

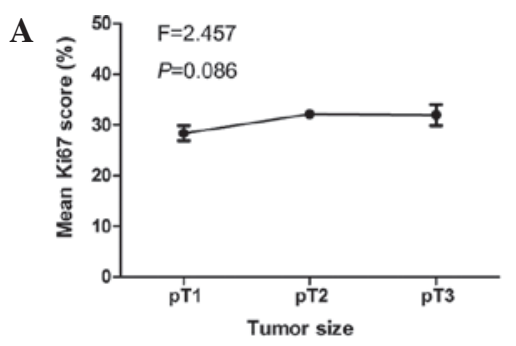

D

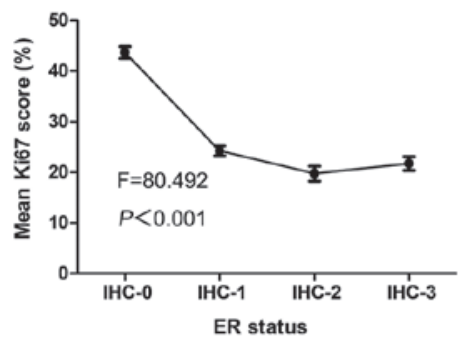

G

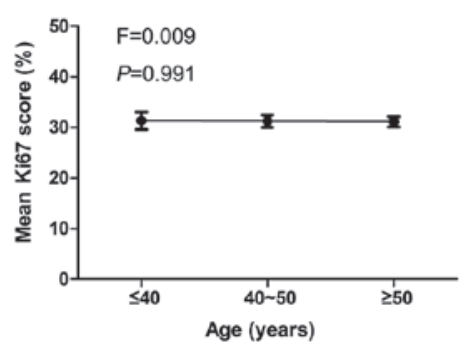

B

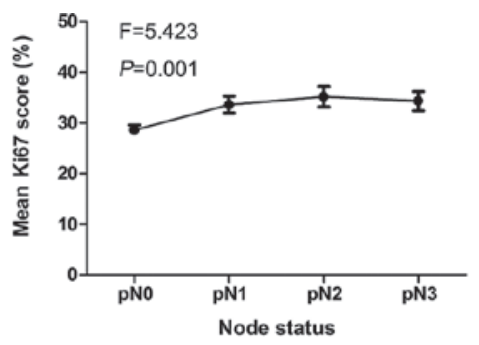

$\mathbf{E}$

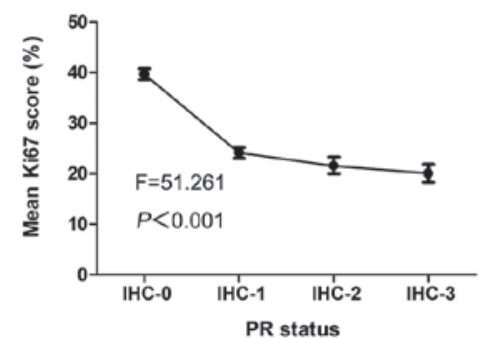

H

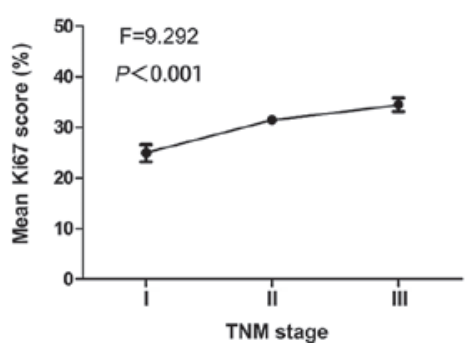

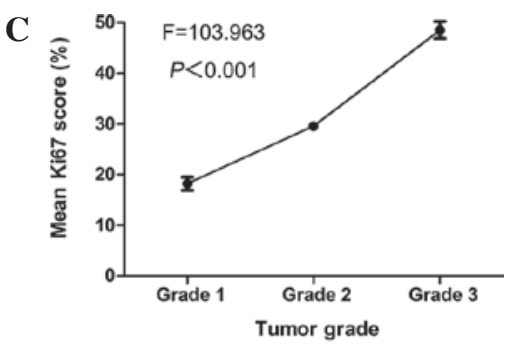

$\mathbf{F}$

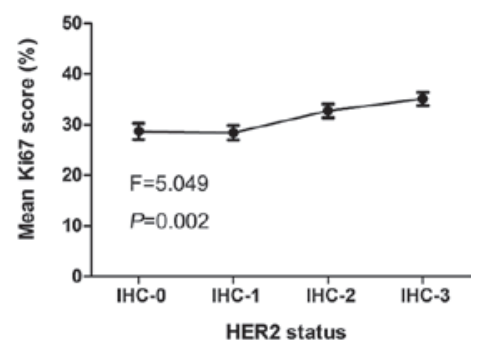

I

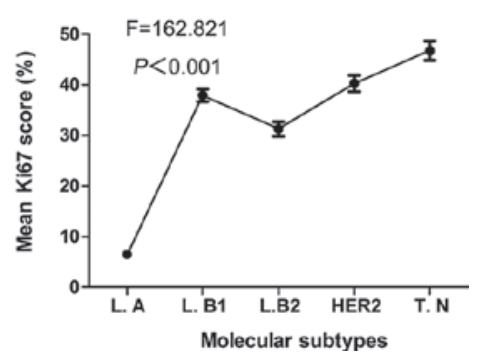

Figure 2. Correlation tendencies between mean Ki67 scores and other clinicopathological parameters. Mean Ki67 scores and correlation with (A) tumor size, (B) lymph node status, (C) tumor grade, (D) ER status, (E) PR status, (F) HER2 status, (G) age, (H) TNM stage and (I) molecular subtypes. ER, estrogen receptor; PR, progesterone receptor; HER2, human epidermal growth factor receptor 2; IHC, immunohistochemical stage; TNM, tumor-node-metastasis; L.A, luminal A; L.B1, luminal B (high Ki67); L.B2, luminal B (HER2-positive); HER2, HER2-positive (non-luminal); T.N, triple-negative. 


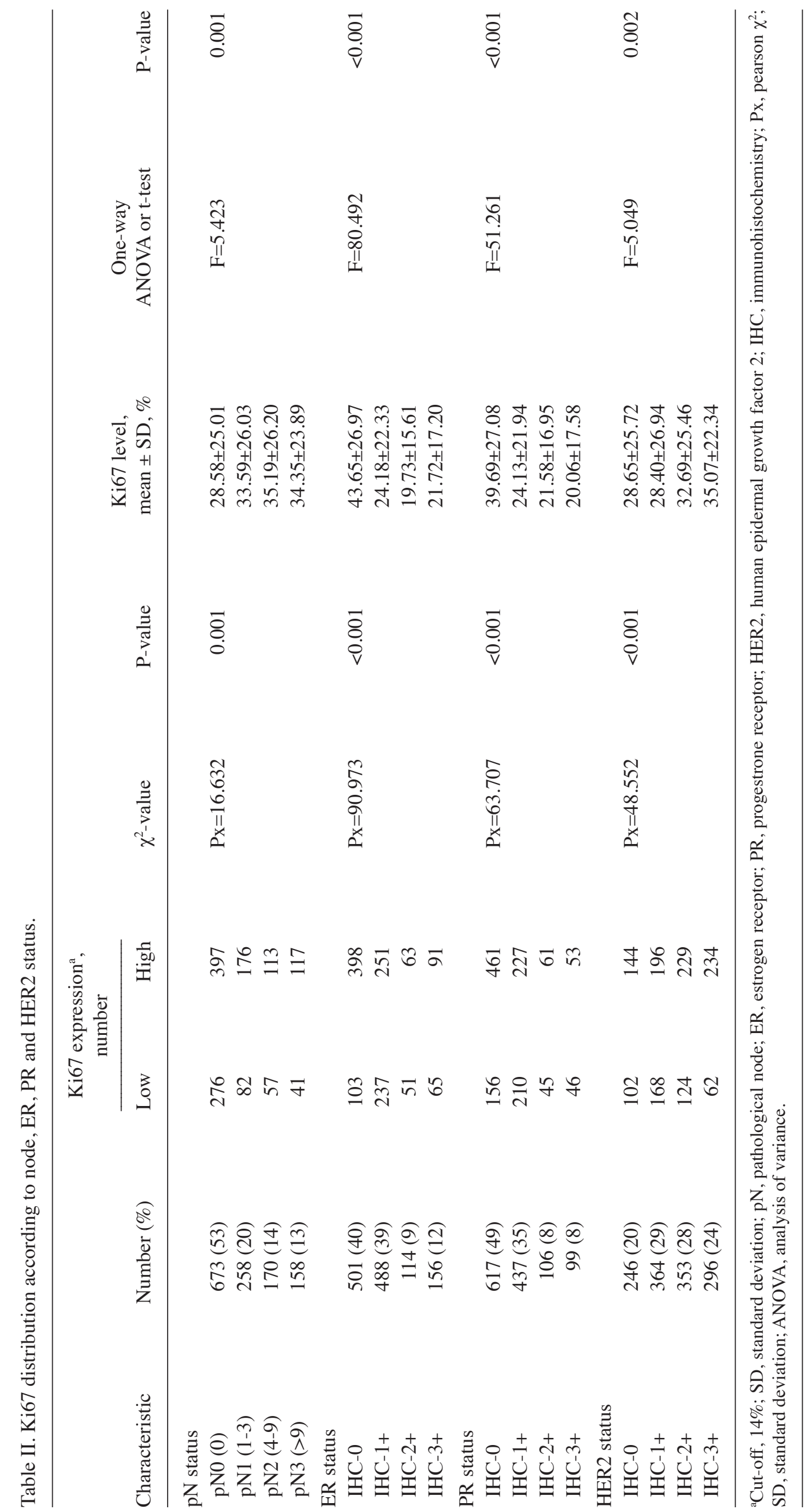


Table III. Ki67 expression in different molecular breast cancer subtypes.

\begin{tabular}{|c|c|c|c|c|c|}
\hline Variables & $\mathrm{T} \leq 2 \mathrm{~cm}$ & $\mathrm{~T}>2 \mathrm{~cm}$ & Node-negative & Node-positive & Total \\
\hline \multicolumn{6}{|l|}{ Luminal A } \\
\hline Low Ki67 expression ${ }^{\text {a }}$ & 88 & 220 & 188 & 120 & 308 \\
\hline High Ki67 expression $^{a}$ & 0 & 0 & 0 & 0 & 0 \\
\hline Ki67 level $(\%)^{\mathrm{b}}$ & $5.55 \pm 3.21$ & $6.85 \pm 3.23$ & $6.16 \pm 3.35$ & $6.97 \pm 3.09$ & $6.48 \pm 3.27$ \\
\hline \multicolumn{6}{|l|}{ Luminal B (high ki67) } \\
\hline Low Ki67 expression ${ }^{\mathrm{a}}$ & 0 & 0 & 0 & 0 & 0 \\
\hline High Ki67 expression $^{\mathrm{a}}$ & 60 & 214 & 138 & 136 & 274 \\
\hline Ki67 level $(\%)^{\mathrm{b}}$ & $41.17 \pm 22.59$ & $37.03 \pm 18.92$ & $36.49 \pm 19.64$ & $39.41 \pm 19.94$ & $37.94 \pm 19.81$ \\
\hline \multicolumn{6}{|c|}{ Luminal B (HER2-positive) } \\
\hline Low Ki67 expression ${ }^{\text {a }}$ & 20 & 34 & 27 & 27 & 54 \\
\hline High Ki67 expression $^{a}$ & 38 & 119 & 72 & 85 & 157 \\
\hline Ki67 level $(\%)^{\mathrm{b}}$ & $27.91 \pm 21.51$ & $32.60 \pm 21.22$ & $29.92 \pm 20.84$ & $32.54 \pm 21.81$ & $31.31 \pm 21.35$ \\
\hline \multicolumn{6}{|c|}{ HER2-positive (non-luminal) } \\
\hline Low Ki67 expression ${ }^{\mathrm{a}}$ & 8 & 32 & 21 & 19 & 40 \\
\hline High Ki67 expression ${ }^{a}$ & 39 & 151 & 92 & 98 & 190 \\
\hline Ki67 level $(\%)^{\mathrm{b}}$ & $43.49 \pm 22.63$ & $39.47 \pm 24.34$ & $37.59 \pm 23.70$ & $42.90 \pm 24.11$ & $40.29 \pm 24.00$ \\
\hline \multicolumn{6}{|l|}{ Triple negative } \\
\hline Low Ki67 expression ${ }^{\mathrm{a}}$ & 13 & 41 & 40 & 14 & 54 \\
\hline High Ki67 expression $^{a}$ & 29 & 153 & 95 & 87 & 182 \\
\hline Ki67 level $(\%)^{\mathrm{b}}$ & $41.55 \pm 30.32$ & $47.93 \pm 28.46$ & $43.17 \pm 29.79$ & $51.63 \pm 26.90$ & $46.79 \pm 28.83$ \\
\hline \multicolumn{6}{|c|}{ Statistical analysis (P-value) } \\
\hline$\chi^{2}$ test & $<0.001$ & 0.001 & 0.001 & 0.001 & 0.001 \\
\hline One-way ANOVA & $<0.001$ & 0.001 & 0.001 & 0.001 & 0.001 \\
\hline Dunnett's T3 test ${ }^{\mathrm{c}}$ & 1 & 0.020 & 0.654 & 0.121 & 0.081 \\
\hline
\end{tabular}

${ }^{\mathrm{a}} \mathrm{Cut}-\mathrm{off}, 14 \%$; ${ }^{\mathrm{b}}$ data are expressed as the mean \pm standard deviation; ${ }^{\mathrm{c}}$ comparison between HER2-positive (non-luminal) and triple-negative subtypes. HER2, human epidermal growth factor receptor 2; T, tumor size; ANOVA, analysis of variance.

The mean Ki67 score was $31.22 \%$ (median, 25\%; range, 0-91\%). Overall, the Ki67 scores were as follows: $\leq 15 \%$ in 500 cases; $>15 \%$ but $\leq 30 \%$ for 281 cases; $>30 \%$ but $\leq 45 \%$ in 93 cases; $>45 \%$ but $\leq 60 \%$ for 221 cases; $>60 \%$ but $\leq 75 \%$ in 27 cases; and $>75 \%$ in 137 cases (Fig. $1 \mathrm{~A}$ ). The clinicopathological characteristics, mean Ki67 scores and Ki67 expression levels are listed in Table I.

Of the 1,259 eligible patients, 308 (24\%) were classified with a luminal A subtype, 274 (22\%) with luminal B (high Ki67), 211 (17\%) with luminal B (HER2-positive), 230 (18\%) with HER2-positive (non-luminal) and 236 (19\%) with a triple-negative subtype. The mean Ki67 scores of these subtypes were $6.48,37.94,31.31,40.29$ and $46.79 \%$, respectively. Significant differences were identified between the Ki67 scores of different BC subtypes (Fig. 1B).

Association between Ki67 and other clinicopathological parameters. As a continuous variable, statistically significant differences were observed between the mean Ki67 scores and the lymph node status, tumor grade, ER, PR and HER2 status, clinical stage and molecular subtypes $(\mathrm{P}<0.001$; Fig. 2). When Ki67 was categorized into high $(>14 \%)$ and low $(\leq 14 \%)$ level groups, the $\chi^{2}$ test was used to verify these results. No statistically significant associations were identified between the mean Ki67 scores and age $(\mathrm{P}=0.991)$ or tumor size $(\mathrm{P}=0.086)$. When $\mathrm{Ki67}$ was a categorized variable, a statistically significant association was revealed between Ki67 expression and tumor size $(\mathrm{P}=0.002)$. The data are presented in Table II. Fig. 2 demonstrates the correlation tendency between the mean Ki67 scores and the clinicopathological parameters of tumor size, lymph node status, tumor grade, ER, PR and HER2 status, age, TNM stage and molecular subtype.

Ki67 expression in molecular subtypes with various lymph node statuses or tumor sizes. Ki67 expression in the five molecular subtypes and its association with the various lymph node statuses or tumor sizes are listed in Table III. Statistically significant differences were identified between BC subtypes with different Ki67 scores and the various lymph node statuses and tumor sizes. In addition, the mean Ki67 scores were found to be higher in the triple-negative subtype compared with the HER2-positive (non-luminal) subtype. However, no statistically significant differences were evident between the mean Ki67 scores in the HER2-positive (non-luminal) and triple-negative subtypes, with the exception of patients with tumors with a size of $>2 \mathrm{~cm}(\mathrm{P}=0.02$; Fig. 1B-D). 


\section{Discussion}

To the best of our knowledge, this is the first study to describe the distribution of Ki67 expression in BC. However, Ki67 expression levels were previously reported in several small-sample BC studies $(13,21,22)$. Haroon et al (13) identified that $239.8 \%$ (78/194) of the BC patients included in the study exhibited a low Ki67 expression (cut-off, 15\%), while Fasching et al observed the same in 29.3\% (162/552; cut-off, $13 \%$ ) of the included patients (21). Furthermore, $\sim 36.2 \%(471 / 1,302)$ of invasive ductal BC patients and 59.7\% (237/397) of invasive lobular BC patients demonstrated a low Ki67 expression (cut-off, 14\%) in a study by Heusinger et al (22). Although the analysis of Ki67 expression may differ from previous studies, the present study demonstrated similar findings, with $36.2 \%(456 / 1,259)$ of patients with a low Ki67 expression (cut-off, 15\%). The findings of the present study also indicated that the distribution of Ki67 expression in a Chinese cohort of $\mathrm{BC}$ cases may be the same as that in other countries.

A number of previous studies have investigated the correlation between Ki67 and other clinicopathological parameters (13-15); however, the findings were controversial. A study that included a cohort of Pakistani patients revealed a significant association between Ki67 expression and tumor grade, PR, HER2 and lymph node status. However, no correlation was identified between the ER status and tumor size (13). The earliest study conducted in the United Kingdom, demonstrated a significant association between the Ki67 index and the histological grade, size and type of the tumors (14). However, these studies included only a small number of samples. The present population-based study revealed that Ki67 was significantly associated with all the clinicopathological parameters, with the exception of age. In accordance with previous studies, the present study confirmed the importance of the Ki67 level in predicting the prognosis of $\mathrm{BC}$ (23-26).

The results of the present study also identified marked differences between the Ki67 scores and the levels of the ER, PR and HER2. Higher levels of ER and PR were correlated with declining Ki67 scores, while higher levels of HER2 were associated with increasing Ki67 scores. These results indicated that there was an increased proliferative activity in the BC cells with lower levels of ER and PR, or higher levels of HER2, and that Ki67 is an accurate biomarker that reflects tumor cellular proliferate activity. Furthermore, the results demonstrated that the Ki67 score increased with increasing tumor size in the early stages of BC. However, when the BC progressed to a certain stage, the Ki67 score did not increase accordingly. This indicated that the proliferative activity increases with the progression of a tumor to a certain stage, at which it no longer significantly changes. This may be due to insufficient blood supply and nutrition, which is unable to support tumor growth after a certain point.

A previous study also identified higher Ki67 expression levels in triple-negative and HER2-positive subtypes compared with the luminal subtypes (27). However, whether the levels of Ki67 are highest in the triple-negative or the HER2-positive subtype required further investigation. In the present study, the expression of molecular subtypes with various tumor sizes and lymph node statuses were also identified. The results revealed that the mean Ki67 scores were not significantly different between the HER2-positive (non-luminal) and triple-negative subtypes, with the exception of patients with a tumor size of $>2 \mathrm{~cm}$. This indicated the presence of stronger proliferative activity in the triple-negative subtype compared with the HER2-positive (non-luminal) subtype, with regard to patients with a tumor size of $>2 \mathrm{~cm}$ BC patients.

The present study also has certain limitations. The immunohistochemical approaches that were used had limited technical reproducibility, subjective interpretation and qualitative readouts. In addition the samples and data obtained for the study were from different hospitals and pathology laboratories, which may have lead to specific biases. Nevertheless, this population-based study from central China demonstrated the correlation between Ki67 and other clinicopathological parameters in invasive BC cases. Furthermore, to the best of our knowledge, this study contained the greatest number of samples used in China and in other counties to investigate the distribution of $\mathrm{Ki} 67$ in BC patients.

In conclusion, in the present study a significant correlation was identified between Ki67 and ER, PR, and HER2 status, tumor size, lymph node status, tumor grade and molecular subtypes in invasive $\mathrm{BC}$, which indicates that Ki67 presents an important biomarker. Thus, analysis of Ki67 expression may be useful in clinical practice and may present an option for the personalized treatment of BC patients in the future.

\section{Acknowledgements}

This study was supported by grants from the National Science Foundation of China (nos. 81201196, 81302314 and 81230031), the Fundamental Research Funds for the Central Universities (no. 121004), the Natural Science Foundation of Hubei Province, China (nos. 301130851, 2011CBD489 and 2013CFB374), the Research Foundation of Public Health Bureau of Hubei Province (nos. JS-2011018, JX4B19 and JX3A14) and the National Science and Technology Major Project of the Ministry of Science and Technology of China (no. 2012YQ16020306).

\section{References}

1. Jemal A, Bray F, Center MM, Ferlay J, Ward E and Forman D: Global cancer statistics. CA Cancer J Clin 61: 69-90, 2011.

2. Gerdes J, Schwab U, Lemke H and Stein H: Production of a mouse monoclonal antibody reactive with a human nuclear antigen associated with cell proliferation. Int J Cancer 31: 13-20, 1983.

3. Gerdes J, Lemke H, Baisch H, Wacker HH, Schwab U and Stein H: Cell cycle analysis of a cell proliferation-associated human nuclear antigen defined by the monoclonal antibody Ki-67. J Immunol 133: 1710-1715, 1984.

4. Gerdes J, Li L, Schlueter C, et al: Immunobiochemical and molecular biologic characterization of the cell proliferationassociated nuclear antigen that is defined by monoclonal antibody Ki-67. Am J Pathol 138: 867-873, 1991.

5. de Azambuja E, Cardoso F, de Castro G Jr, et al: Ki-67 as prognostic marker in early breast cancer: a meta-analysis of published studies involving 12,155 patients. Br J Cancer 96: 1504-1513, 2007.

6. Kontzoglou K, Palla V, Karaolanis G, et al: Correlation between Ki67 and breast cancer prognosis. Oncology 84: 219-225, 2013.

7. Jones RL, Salter J, A'Hern R, et al: Relationship between oestrogen receptor status and proliferation in predicting response and long-term outcome to neoadjuvant chemotherapy for breast cancer. Breast Cancer Res Treat 119: 315-323, 2010. 
8. Ellis MJ, Coop A, Singh B, et al: Letrozole inhibits tumor proliferation more effectively than tamoxifen independent of HER $1 / 2$ expression status. Cancer Res 63: 6523-6531, 2003.

9. Dowsett M, Smith IE, Ebbs SR, et al; IMPACT Trialists: Short-term changes in Ki-67 during neoadjuvant treatment of primary breast cancer with anastrozole or tamoxifen alone or combined correlate with recurrence-free survival. Clin Cancer Res 11: 951s-958s, 2005.

10. Dowsett M, Nielsen TO, A'Hern R, et al; International Ki-67 in Breast Cancer Working Group: Assessment of Ki67 in breast cancer: recommendations from the International Ki67 in Breast Cancer Working Group. J Natl Cancer Inst 103: 1656-1664, 2011

11. Cheang MC, Chia SK, Voduc D, et al: Ki67 index, HER2 status, and prognosis of patients with luminal B breast cancer. J Natl Cancer Inst 101: 736-750, 2009.

12. Goldhirsch A, Wood WC, Coates AS, Gelber RD, Thürlimann B and Senn HJ; Panel Members: Strategies for subtypes - dealing with the diversity of breast cancer: highlights of the St. Gallen International Expert Consensus on the Primary Therapy of Early Breast Cancer 2011. Ann Oncol 22: 1736-1747, 2011.

13. Haroon S, Hashmi AA, Khurshid A, et al: Ki67 index in breast cancer: correlation with other prognostic markers and potential in pakistani patients. Asian Pac J Cancer Prev 14: 4353-4358, 2013.

14. Pinder SE, Wencyk P, Sibbering DM, et al: Assessment of the new proliferation marker MIB1 in breast carcinoma using image analysis: associations with other prognostic factors and survival. Br J Cancer 71: 146-149, 1995.

15. Midulla C, De Iorio P, Nagar C, et al: Immunohistochemical expression of p53, nm23-HI, Ki67 and DNA ploidy: correlation with lymph node status and other clinical pathologic parameters in breast cancer. Anticancer Res 19: 4033-4037, 1999.

16. No authors listed: The World Health Organization. Histological typing of breast tumors. Neoplasma 30: 113-123, 1983.

17. Robbins P, Pinder S, de Klerk N, et al: Histological grading of breast carcinomas: a study of interobserver agreement. Hum Pathol 26: 873-879, 1995.

18. Edge SB, Byrd DR, Compton CC, et al (eds.): AJCC Cancer Staging Manual. 7th edition. Springer, New York, NY, USA, 2009.
19. Hammond ME, Hayes DF, Dowsett M, et al: American Society of Clinical Oncology/College Of American Pathologists guideline recommendations for immunohistochemical testing of estrogen and progesterone receptors in breast cancer. J Clin Oncol 28: 2784-2795, 2010

20. Wolff AC, Hammond ME, Hicks DG, et al; American Society of Clinical Oncology; College of American Pathologists: Recommendations for human epidermal growth factor receptor 2 testing in breast cancer: American Society of Clinical Oncology/College of American Pathologists clinical practise guideline update. J Clin Oncol 31: 3997-4013, 2013.

21. Fasching PA, Heusinger K, Haeberle L, et al: Ki67, chemotherapy response, and prognosis in breast cancer patients receiving neoadjuvant treatment. BMC Cancer 11: 486, 2011.

22. Heusinger K, Jud SM, Haeberle L, et al: Association of mammographic density with the proliferation marker Ki-67 in a cohort of patients with invasive breast cancer. Breast Cancer Res Treat 135: 885-892, 2012

23. Ferguson NL, Bell J, Heidel R, et al: Prognostic value of breast cancer subtypes, Ki-67 proliferation index, age, and pathologic tumor characteristics on breast cancer survival in Caucasian women. Breast J 19: 22-30, 2013.

24. Reyal F, Hajage D, Savignoni A, et al: Long-term prognostic performance of Ki67 rate in early stage, $\mathrm{pT} 1-\mathrm{pT} 2$, pN0, invasive breast carcinoma. PLoS One 8: e55901,2013.

25. Yerushalmi R, Woods R, Ravdin PM, Hayes MM and Gelmon KA: Ki67 in breast cancer: prognostic and predictive potential. Lancet Oncol 11: 174-183, 2010.

26. Kontzoglou K, Palla V, Karaolanis G, et al: Correlation between Ki67 and breast cancer prognosis. Oncology 84: 219-225, 2013.

27. Mitrović O, Čokić V, Đikić D, et al: Correlation between ER, PR, HER-2, Bcl-2, p53, proliferative and apoptotic indexes with HER-2 gene amplification and TOP2A gene amplification and deletion in four molecular subtypes of breast cancer. Target Oncol 9: 367-379, 2014. 